\title{
MONOGRÁFICO: La red INNOVAGRO y la bioeconomía
}

\author{
Martha Escalante ${ }^{1}$ y Lucía Reguillo ${ }^{2}$
}

Autor de Correspondencia: opi02.op@ceia3.es

\section{Resumen:}

Nos encontramos ante un escenario cambiante lleno de desafíos globales con interconexión entre países. La dependencia de recursos fósiles y el agotamiento de los recursos naturales, configuran un panorama crítico que requiere de manera urgente que se adopten medidas a nivel de la sociedad y de los sectores público y privado. En este sentido, la bioeconomía, es una herramienta multidisciplinar y colaborativa para abordar los citados desafíos globales. Para su puesta en práctica, afrontada desde distintas perspectivas, la cooperación a nivel internacional de instituciones con objetivos comunes supone un importante paso para ser parte de tan importante y necesario cambio. En este contexto, se presenta este monográfico, que tiene como objetivo exponer el rol de la Red INNOVAGRO y ejemplificar su acción mediante posters, con la información útil y simplificada de los distintos miembros de la Red en el ámbito de la bioeconomía.

Palabras clave: competitividad, desarrollo sustentable, bioeconomía, Iberoamérica, cooperación

\section{MONOGRAPH: The INNOVAGRO Network and the Bioeconomy}

\author{
Martha Escalante ${ }^{1}$ y Lucía Reguillo 2
}

\begin{abstract}
:
We are now facing a changing scenario full of global challenges characterised by an interconnection between countries. Dependence on fossil resources and the depletion of natural ones give rise to a critical panorama that urgently requires action by society and the public and private sectors. In this regard, the Bioeconomy, constitutes a multidisciplinary and collaborative tool to address these global challenges. In order to put it into practice from different perspectives, cooperation at the level of international institutions sharing common objectives is an important step towards this important and necessary shift. This monograph is presented in this context, aiming to make known the role of the INNOVAGRO Network and explain its actions through posters featuring useful and simplified information from the various members of the Network in the field of the Bioeconomy.
\end{abstract}

Keywords: Competitiveness, sustainable development, bioeconomy, Latin America, cooperation

1 Red INNOVAGRO, Instituto Interamericano de Cooperación para la Agricultura (IICA), (México), martha.escalante@iica.int

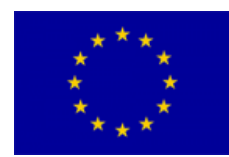

UNION EUROPEA

PROYECTO COFINANCIADO POR EL FONDO EUROPEO DE DESARROLLO REGIONA

Una manera de hacer Europa

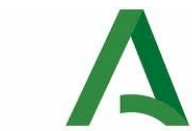

Junta de Andalucía
Andalucía

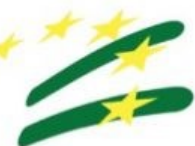

se mueve con Europa 
2 Campus de Excelencia Internacional Agroalimentario (ceiA3), (España), opi02.op@ceia3.es

\section{LA RED INNOVAGRO Y LA BIOECONOMÍA}

En la actualidad, el mundo se enfrenta a una serie de desafíos globales interconectados, tales como la dependencia de recursos fósiles, la seguridad alimentaria, el crecimiento demográfico, la globalización, los efectos del cambio climático, la pérdida de biodiversidad y la escasez de recursos naturales. Ante este escenario, es vital repensar nuestros modelos de producción y nuestros paradigmas de desarrollo, desde lo local hasta lo global que permitan garantizar sistemas agroalimentarios sostenibles a medio y largo plazo. La bioeconomía y su carácter transversal se configura como respuesta a un entorno actualmente preocupante y como una herramienta multidisciplinar y colaborativa para abordar los retos globales.

Ante el escenario anteriormente citado, se plantea la necesidad de superar la antigua visión del sector agropecuario como generador de bienes primarios y ver a la agricultura como la industria de la biomasa y el actor central de los nuevos tiempos en sociedades que aspiran a ser más eficientes en el uso de los recursos naturales y menos dependientes de los recursos fósiles. Para ello se requieren modelos productivos climáticamente responsables, en los que la bioeconomía expresa en buena medida esa visión transformadora para promover el desarrollo agrícola sostenible e inteligente, partiendo de la amplia base de recursos biológicos que poseemos (FAO. 2019).

En Iberoamérica diferentes países han apostado por poner en marcha iniciativas y políticas regionales y nacionales para promover la innovación orientada hacia una producción sostenible y la economía circular.

Con un carácter más global el Instituto Interamericano de Cooperación para la Agricultura (IICA) en su plan a mediano plazo 2018-2022 incluye como primer programa eje, el de "Bioeconomía y Desarrollo Productivo". 
Por otro lado, en Europa, tanto a nivel comunitario como nacional o regional, se ha destacado la importancia y necesidad de la bioeconomía en el contexto actual. En este sentido, basándose en el Plan de Acción, 2018 publicado por la Comisión Europea, se ha lanzado una estrategia de bioeconomía que pivota sobre los pilares de dicho plan de acción: (i) fortalecer el sector con potencial en el desarrollo de productos bioeconómicos, (ii) una rápida implementación de bioeconomía local a lo largo de toda Europa y (iii) conocer los límites de la bioeconomía.

Por otro lado, según datos del Ministerio de Agricultura, Pesca y Alimentación, el peso del sector agroalimentario español en la Unión Europea aumentó en 2019 un 9,1\% en comparación con el año anterior, siendo el primer productor en cítricos y aceite de oliva y quinto país que más aporta en valor bruto a la Unión Europea. España, por tanto, es un país clave en los compromisos internacionales adoptados en el marco de los Objetivos de Desarrollo Sostenible de la Organización de las Naciones Unidas, en los que el sector agroalimentario y pesquero son piezas clave para el cumplimiento de gran parte de ellos.

Entidades públicas como el Campus de Excelencia Internacional Agroalimentario, ceiA3, resultan estratégicas como así lo describen diversos documentos para el desarrollo de los nuevos modelos resilientes en el sector agroalimentario.

Para la Red de Gestión de la Innovación en el Sector Agroalimentario (Red INNOVAGRO) como plataforma que impulsa la innovación en los ecosistemas agroalimentarios de las economías rurales de los 15 países (Argentina, Bolivia, Brasil, Chile, Colombia, Costa Rica, El Salvador, España, Estados Unidos de América, Holanda, México, Nicaragua y República Dominicana) representados a través de sus 72 miembros del sector público y privado (Centros de investigación, instituciones del sector público, universidades, empresas, fundaciones, redes y sistemas regionales; organizaciones de la sociedad civil, organismos internaciones e instituciones 
financieras), su objetivo es fortalecer la productividad, la competitividad y el desarrollo sustentable desde la perspectiva económica, social y ambiental.

Por ello, la bioeconomía es un tema prioritario que tiene sus bases en la innovación, el conocimiento, la transferencia de tecnología, los modelos sostenibles de producción, la seguridad alimentaria, el cuidado de los recursos naturales y el aprovechamiento biológico de éstos para encontrar soluciones innovadoras a los desafíos globales.

Diferentes tópicos de la bioeconomía se han venido tratando en los Encuentros Anuales de la Red, con el propósito de propiciar una reflexión que contribuya al diseño de estrategias y planes de acción para fortalecer los ecosistemas de innovación en los diferentes países. Como ejemplos de dichos encuentros, en 2019 la "Bioeconomía y los ecosistemas de innovación" constituyó el tema central del IX Encuentro de la Red Innovagro y en 2020, el título ha sido "Senderos de innovación para un sector agroalimentario sostenible y resiliente". En ambos, tanto la innovación, la bioeconomía como la sostenibilidad son aspectos protagonistas de dichos encuentros, que consisten en seminarios, rutas de innovación y sesiones de networking que visibilizan la importancia del sector agroalimentario a nivel global y de la necesidad de crear alianzas internacionales que fortalezcan y promuevan los objetivos descritos con anterioridad.

Con objeto de aportar información útil que ejemplifique la cooperación en el marco de la Red INNOVAGRO, a continuación, se muestran los principales miembros de la Red que cuentan con un fuerte alineamiento con la Bioeconomía, y en cuyo contenido se expone una breve introducción de cada uno de ellos, proyectos e iniciativas relevantes y su alineamiento en la temática. 


\begin{tabular}{l|l}
\hline SOCIO DE LA RED INNOVAGRO & PAÍS \\
\hline BIOFÁBRICA S.XXI & MÉXICO \\
\hline BIOKRONE. EXcellence in biotechnology & MÉXICO \\
\hline CeiA3 & ESPAÑA \\
\hline CIMMYT. Centro internacional de mejoramiento de maíz y trigo & MÉXICO \\
\hline CIREN CHILE. Centro internacional de recursos naturales & CHILE \\
\hline CTA Y LA BIOECONOMÍA CIRCULAR. Corporación tecnológica de Andalucía & ESPAÑA \\
\hline $\begin{array}{l}\text { FITTACORI Y LA BIOECONOMÍA. Fundación para el Fomento y Promoción de la } \\
\text { Investigación y Transferencia de Tecnología Agropecuaria de Costa Rica }\end{array}$ & COSTA RICA \\
\hline $\begin{array}{l}\text { FUNICA. Fundación para el desarrollo tecnológico agropecuario y forestal de } \\
\text { Nicaragua }\end{array}$ & NICARAGUA \\
\hline $\begin{array}{l}\text { ICAT/UNAM. Instituto de ciencias aplicadas y tecnología/Universidad Nacional } \\
\text { Autónoma de México }\end{array}$ & MÉXICO \\
\hline \begin{tabular}{ll} 
IICA. Instituto Interamericano de Cooperación para la Agricultura \\
\hline SNIA. Sistema nacional de investigación agrícola
\end{tabular} & COSTA RICA \\
\hline $\begin{array}{l}\text { TECNOVA CENTRO TECNOLÓGICO } \\
\text { UCR. Universidad de Costa Rica }\end{array}$ & MÉXICO \\
\hline UNALM. Universidad Nacional Agraria La Molina & ESPAÑA \\
\hline $\begin{array}{l}\text { AGROPOLO CAMPINAS-BRASIL VER } \\
\text { WUR. Wageningen University Research. }\end{array}$ & COSTA RICA \\
\hline
\end{tabular}




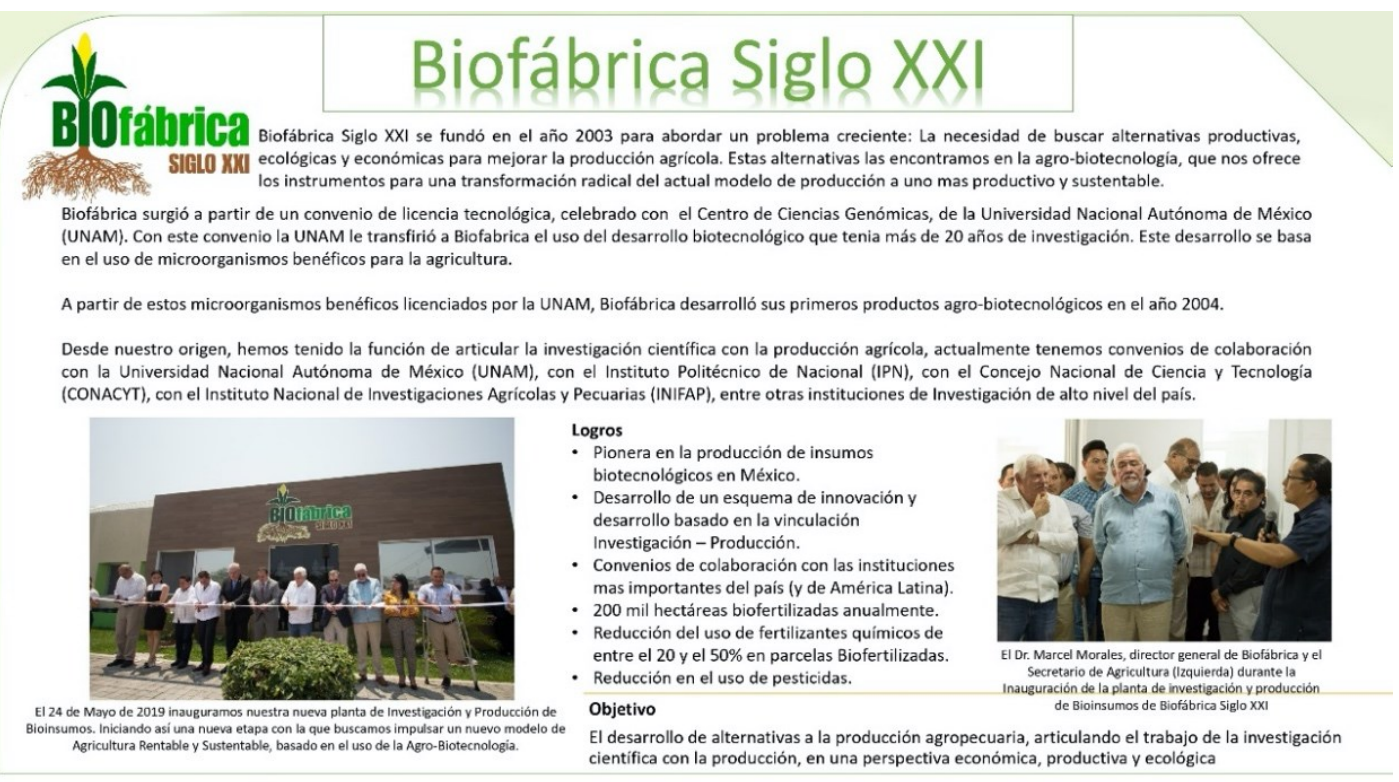

\section{Biofábrica y la Bioeconomía}

El modelo de trabajo de Biofábrica se basa en el impulso de la Bioeconomía

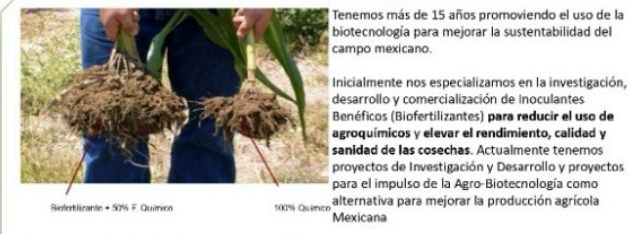

Programa de Agricultura sustentable

Es un programa integrador que vincula al sector académico, gubernamental y productivo para transformar ef sistema de producción agricola en el país. En la primera etapa arrancará en el Estado de Morelos, líder en la producción de diversos cultivos agricolas.

Nuestra propuesta es que es posible incrementar la rentabilidad y sustentabilidad de los cultivos mediante el uso

de la biotecnología y otras prácticas de conservación. Este provecto propon

Disminuir y en algunos casos eliminar el uso de pesticic
Incrementar la rentabilidad de los cultivos mediante:

La disminución de los costos de producción

El incremento en el rendimiento de los cultivos

- Incrementar los ingresos derivados del aumento en la calidad, sanidad e inocuidad de los cultivos

vación y restauración de los suelos agricolas.

Promover la captura de carbono en las parcelas agricolas. Esto permitiri

Disminuir los gases de efecto invernadero

El objetivo es traducir este programa en politcas
cultivos mediante el uso de la biotecnologia
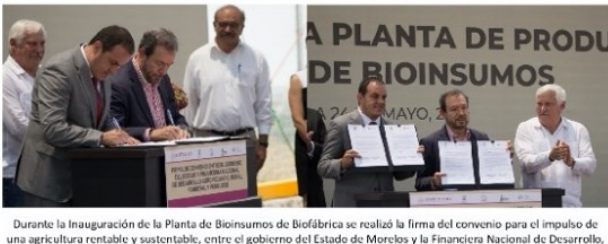

Biotecnologías y prácticas del paquete tecnológico impulsado en el Programa de Agricultura sustentable

- Biofertilizantes (Inoculantes para la promoción del crecimiento

regeneración del suelo)

- Biopesticidas (Inoculantes para el control de plagas y enfermedades) - Bioencapsulados de lenta liberación de fertilizantes. Son tecnologías biológicas basadas en el uso de compuestos orgánicos que secuestran los fertilizantes y los liberan lentamente.

Minerales no procesados químicamente

- Biocarbón

Composta (producida mediante el aprovechamiento de recursos regionales) - Labranza de conservación

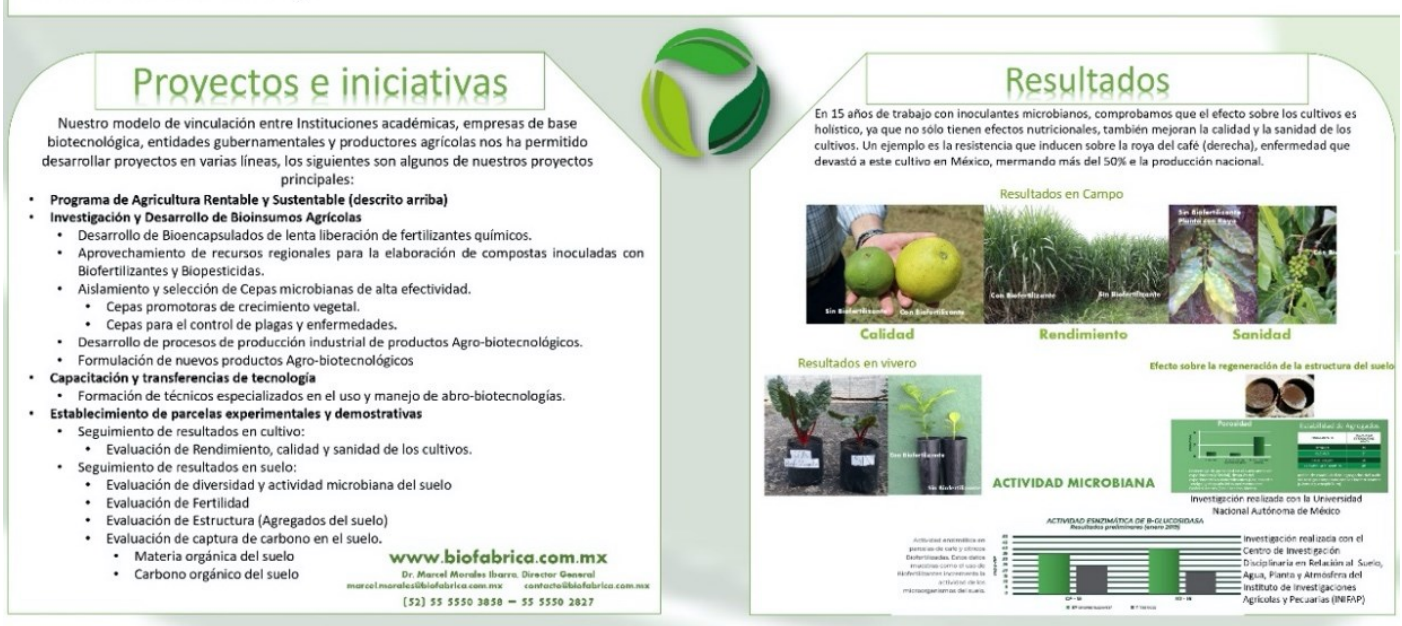

C3-BIOECONOMY, Revista de Investigación y Transferencia en Bioeconomía Circular y Sostenible Nº 1(2020) 


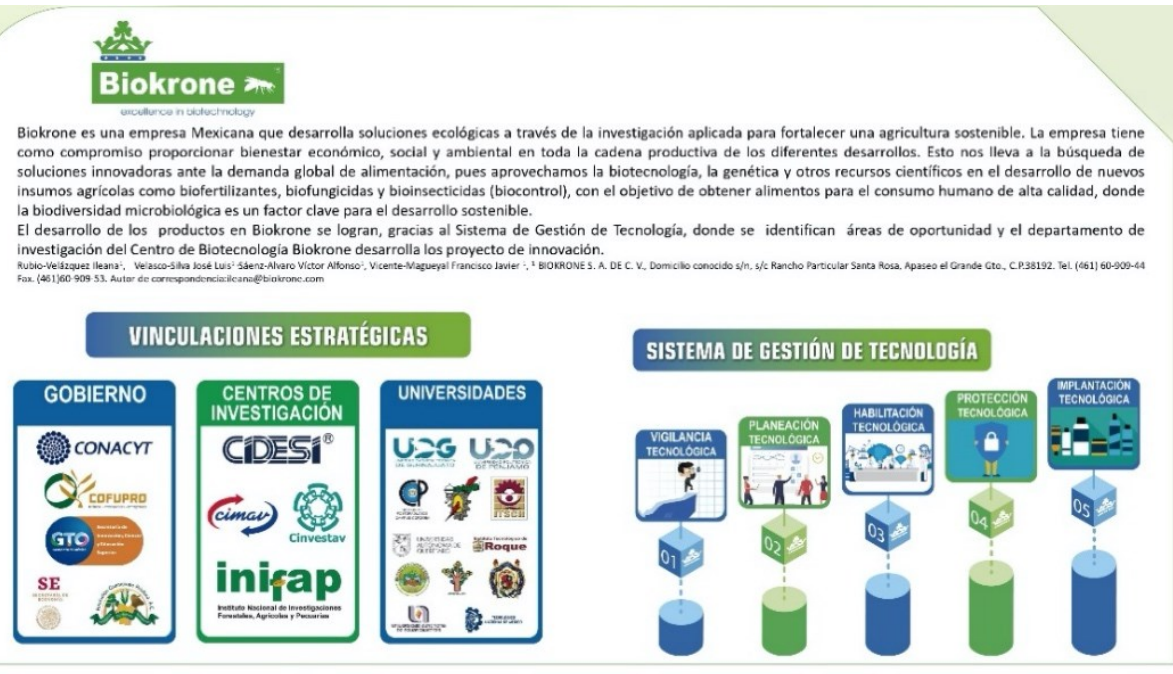

Biokrone y su estrategia en Bioeconomía OBJETIVOS A. Promover y generar soluciones sustentables para
la producción de alimentos, mediante el desarrollo de
productos e ingredientes activos de origen natural. A.Reforzar y promover la capacitación de los
productores y asesoros en el campo, en el uso.

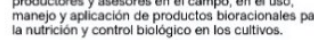
AfFortalecer la vinculación con centros de
investigación, Universidades, Institutos y Empres

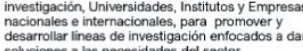
soluciones a las necesidades del sector ULTEERAMIIS EN PROCESO EEONOMMICO BIOKRONE
(iii)
(i) (;)

(ii) (-): (-)

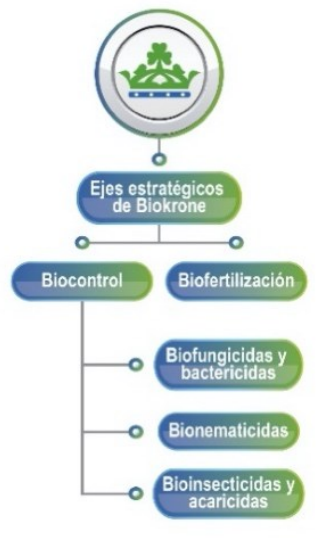

BIOKRONE es una empresa de biotecnologia dedicada producción comercializacion productos $\mathrm{e}$ ingredientes activos de origen natural con la mas alta calidad y pureza, empleando sustentables que aseguran la medio ambiente. Todo este conocimiento es con el objetivo de satisfacer los ejes estrategicos de desarrolio. En comprometidos en atender interactuar con todos los integrantes del proceso económico para atender las necesidades generadas a mejora continua.

Impacto: social, ambiental y económico

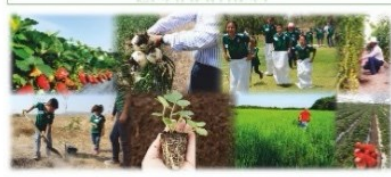

Equipo Biokrone

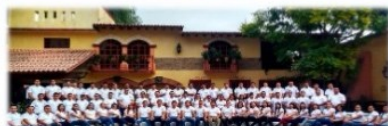

Contacto:

7 /biokrone wentas@biokrone.com

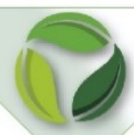

Impactos y resultados

(2) ...... 40 GCADADOS

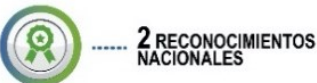

(5) ..... 23\% RENTABILIDAD

(9) -... 2RECONOCDMENTOS

(III) - $20 \%$ CORECIMENTO 


\section{ceiA3}

El Campus de Excelencia Internacional Agroalimentario (ceiA3), integrado por las Universidades de Almería, Cádiz, Córdoba, Huelva y Jaén, con el IFAPA y el CSIC como centros asociados, tiene el reconocimiento internacional como centro de referencia en agroalimentación. El campus cuenta con un órgano de gestión propio, el consorcio ceiA3, que tiene entre sus objetivos promover formación, transferencia, internacionalización e interconexión en el sector agroalimentario de los Investigadores adscritos al campus, y donde destaca la bioeconomía entre sus líneas estratégicas de especialización.

5 UNIVERSIDADES

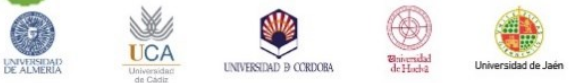

CSIC

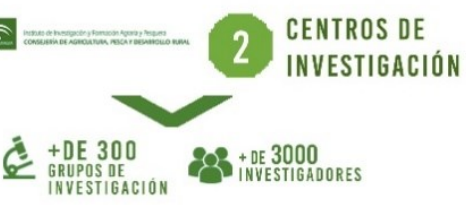

\section{Ejes estrategicos}

- Formación

- Investigación. Innovación y

Transferencia del Conocimiento del Sector Empresarial

- Interacción con el entorno territorial y

la sociedad

- Internacionalización

\section{ceiA3 y la bioeconomía}

Objetivos

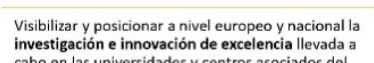
cabo en las universidades y centros asociados del ceiA3.

Reforzar y promover formación y capacitación de los investigadores/as y alumnos y la empleabilidad de egresados de Universidades ceiA 3 .

Promover $y$ fortalecer transferencia de conocimiento $y$ Promover y fortalecer transte
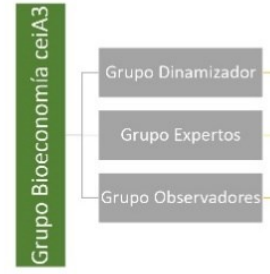

Grupo Expertos
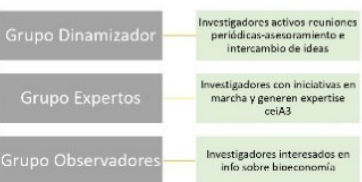

(CEIA3 CLUSter) LIUEAS estrategicas

Alimentación y Salud

Agricultura Sostenible

Biolecrnologia Animal

Biotocnologia Vogetal

Segurldad Allmentarls

Tecnologia Agroalimenteria y
Bloenergia

Economia y Derecho
Agroalimentario

\section{bioeconionity}

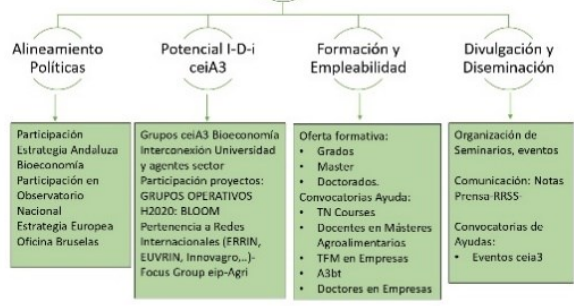

\section{Proyectos e iniciativas}

Investigadores y universidades del ceiA3 han participado en numerosos avalan el potencial del canpus en la materia. A titullo de eja de los proyectos vigentes que cuentan con la participación directa del consorcio ceiA3 junto con sus Grupos de Investigación:

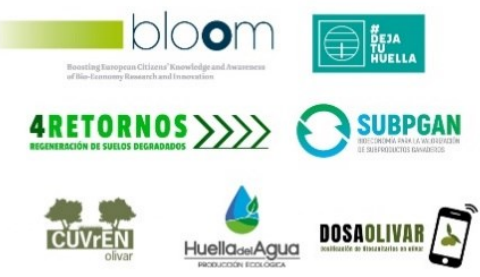

\section{Formación y publicaçiones}

Las universidades del ceiA3 ofertan una amplia variedad de formación de Grado y Máster, contando con las siguientes titulaciones en el ámbito de la

$$
\begin{array}{cccl}
\text { 忙去 } & \checkmark & 23 & \text { GRADOS } \\
\checkmark & \checkmark & 35 & \text { MÁSTERES } \\
\underline{6} & \checkmark & 20 & \text { PROGRAMAS DE } \\
\text { DOCTORADO }
\end{array}
$$

\section{www.ceia3.es}

$$
\text { [I }
$$




\section{VIIMMYT \\ Centro Internacional de Mejoramiento de Maiz y Trigo}

Desde 1966, trabaja en el mundo en desarrollo con el fin de mejorar los medios de vida de las personas y promover sistemas de maíz y de trigo más productivos y sostenibles
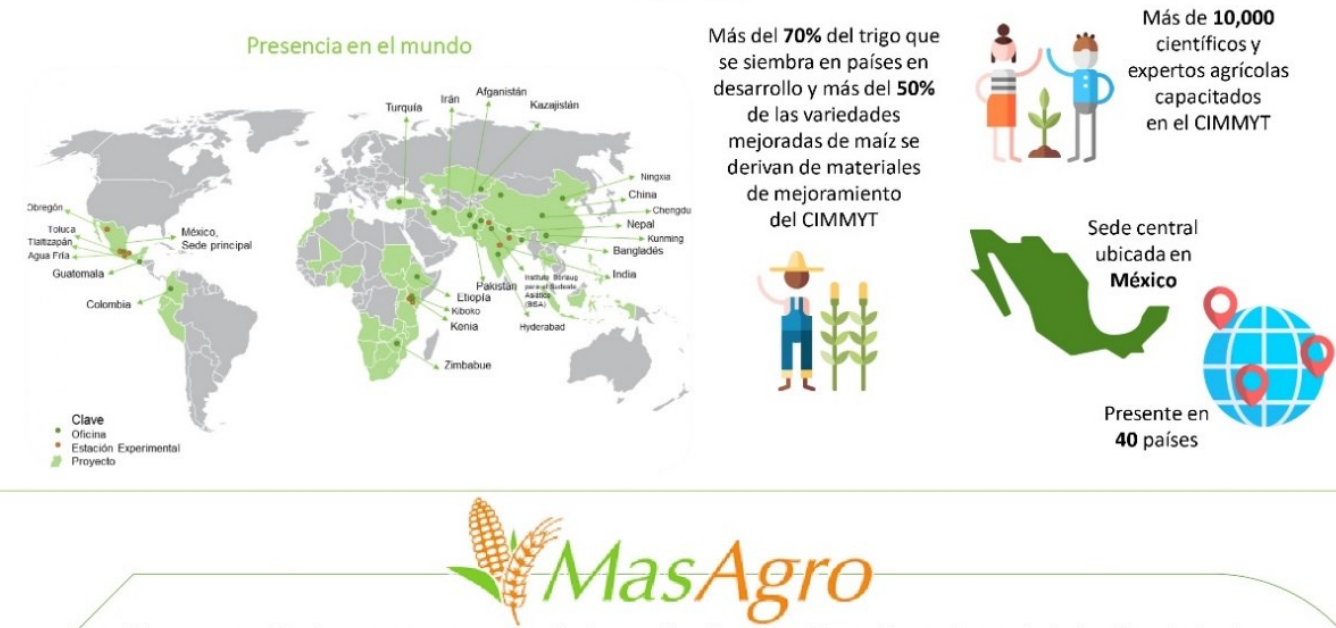

El programa MasAgro, es un proyecto de innovación, investigación y desarrollo rural de la Secretaría de Agricultura y Desarrollo Rural (SADER) y CIMMYT, que promueve prácticas agrícolas sustentables para la generación de sistemas agroalimentarios, incluyentes, sustentables y resilientes.

MasAgro desarrolla investigación y capacidades dirigidas a incrementar la rentabilidad y estabilidad de los rendimientos del maiz y del trigo en México, mediante esquemas de investigación colaborativa, el desarrollo agroalimentario y la difusión de variedades de semillas adaptadas de tecnologías y prácticas agronómicas sostenibles e inteligentes.
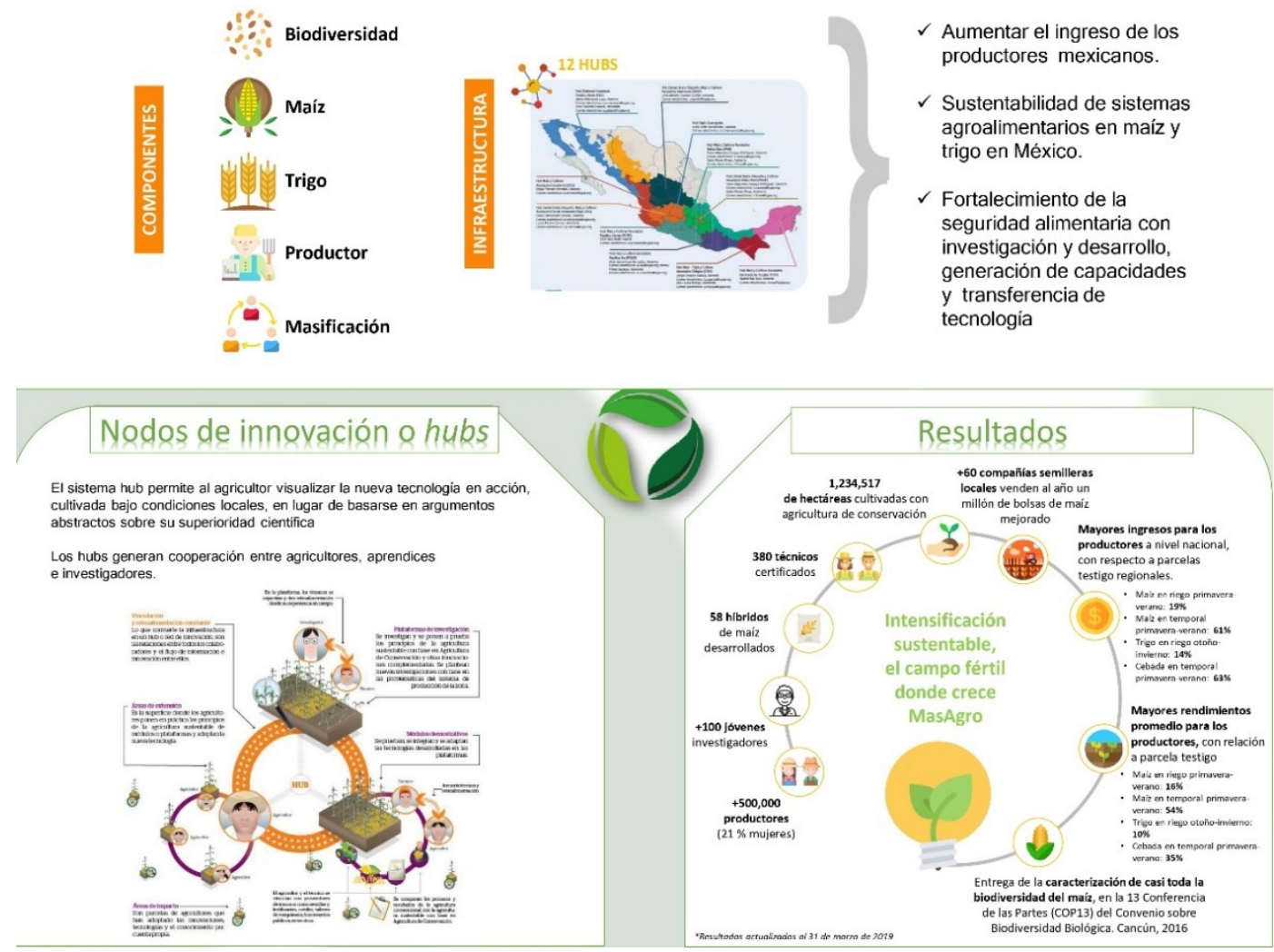


\section{CIREN CHILE}

El Centro de Información de Recursos Naturales, CIREN, es un instituto tecnológico que por más de 30 años ha proporcionado información de valor sobre los recursos naturales y productivos de Chile.

Como servicio de apoyo al Ministerio de Agricultura, trabaja para asegurar la calidad en la provisión de bienes públicos y en la generación de nuevos productos y servicios de alto valor, que contribuyan a la planificación, toma de decisiones y diseño de políticas de desarrollo productivo y de ordenamiento territorial.

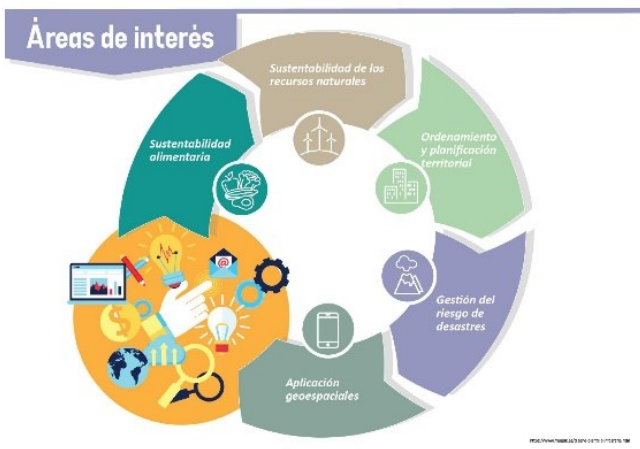

$\checkmark$ Mas de 30 años de experiencia

$\checkmark$ Alto nivel tecnológico y profesional

$\checkmark$ Poseedor de la mayor base de datos georreferenciada de suelos, recursos hidricos y forestales.

$\checkmark$ Realizadores a nivel nacional de catastro

fruticola y de propiedad rural.

$\checkmark$ Premio INNOVAGRO 2018 categoria innovación institucional

\section{CIREN y la Bioecconomía}

Objetivos - Desarrollar un sistema de monitoreo basado en el uso de tecnologias de teledetección y análisis, que permitan la hortícolas en cada región.

- Caracterizar y cuantificar el sector horticola de cada región con el fin de conocer sus fortalezas, debilidades, amenazas y oportunidades, teniendo en cuenta los factores naturales, productivos $y$ comerciales en los que $s$

- Incorporar la plataforma web SITMHOR (Sistema de permite visueritorial para el monitoreo horticola), que permite visualizar, analizar y difundir información productiva horticola, hacien
actualizado.

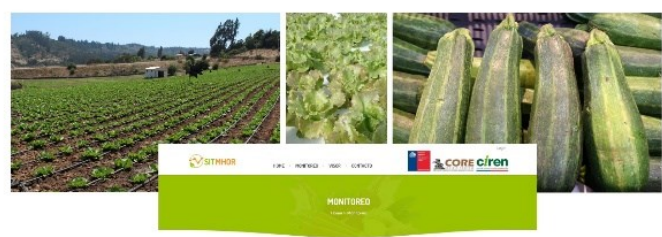

$=$

(2)

$-$

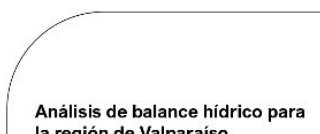
la región de Valparaíso

Este proyecto generó información para contribuir a:

$\checkmark$ Determinar la evolución espaciotemporal de las zonas Valparaiso, la region de 2016.

$\checkmark$ Determinar la relación ofertademanda de agua de riego en el sector agricola.

$\checkmark$ Estimar la variación de los indicadores económicos del dependiendo del suministro agua.
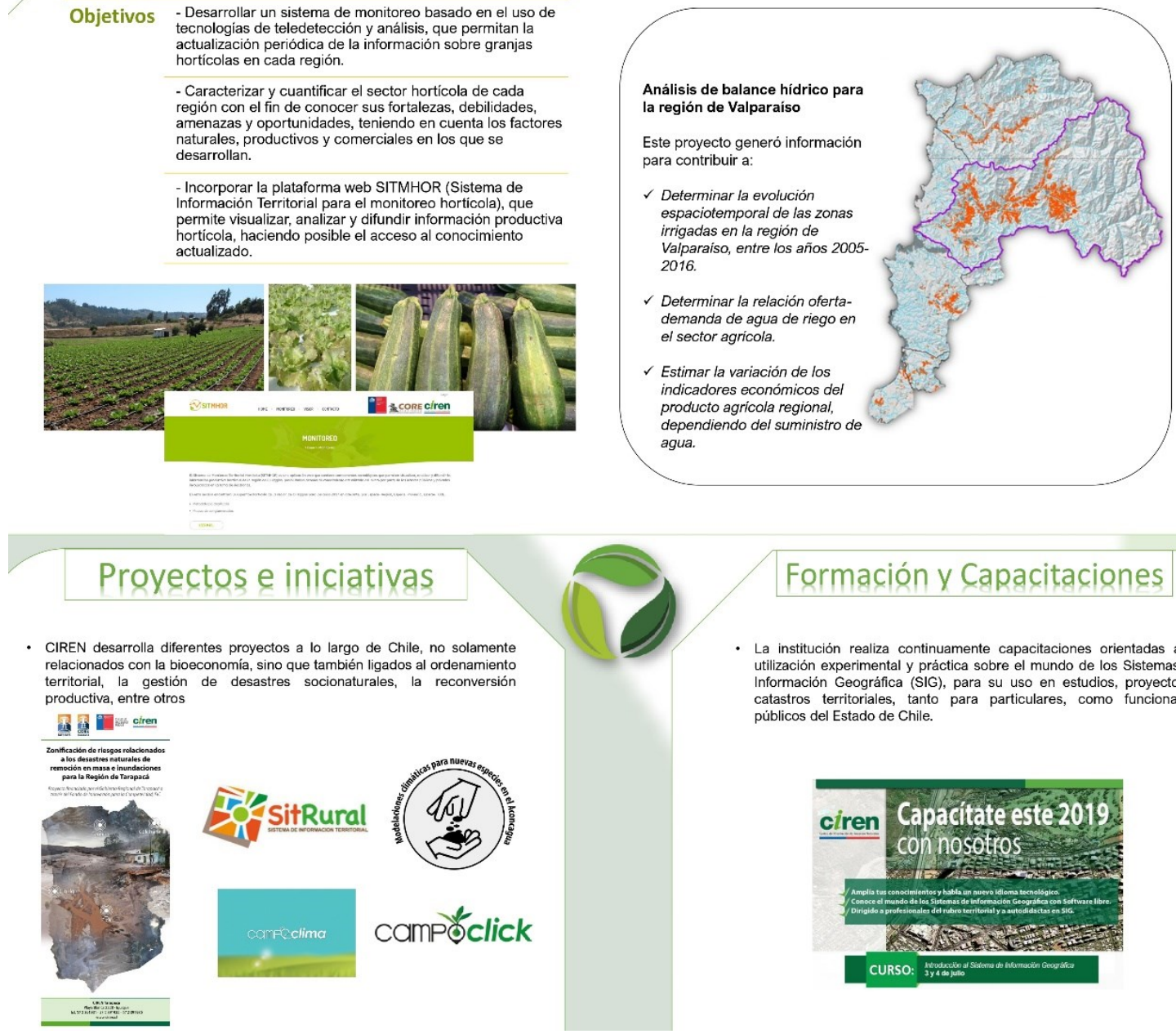

campöclick

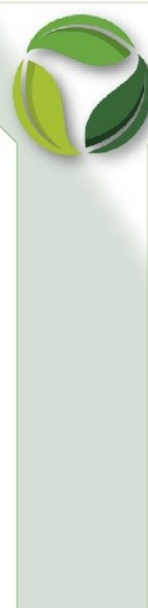

Formación y Capacitaçiones

La institución realiza continuamente capacitaciones orientadas a la utilización experimental y practica sobre el mundo de los Sistemas de Información Geográfica (SIG), para su uso en estudios, proyectos y catastros ternitoriales, tanto para particulares, comos públicos del Estado de Chile.

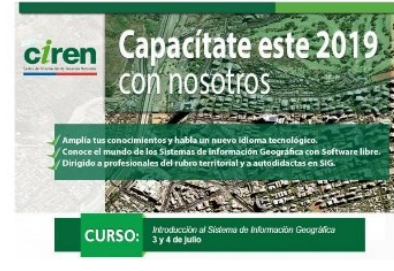

C3-BIOECONOMY, Revista de Investigación y Transferencia en Bioeconomía Circular y Sostenible Nº 1(2020) 


\section{CTA y la bioeconomía circular}

Corporación Tecnológica de Andalucia (CTA) es una fundación privada con más de 160 empresas miembros y más de 13 años de experiencia, impulsada en su origen por la Junta de Andalucia, con gestión privada y dedicada al fomento de la I+D+i empresarial y la transferencia de tecnologia. CTA mantiene una firme apuesta por la bioeconomia circular desde hace varios años. Financia proyectos empresariales de $1+D+i$ con fondos propios en este ámbito, organiza eventos sobre esta temática para movilizar nuevos proyectos e impulsar este sector desde Andalucia y ha asesorado a las Administraciones Públicas en el desarrollo de sus estrategias de bioeconomía y economía circular. Además, ofrece apoyo técnico y legal en procesos de Compra Pública de Innovación "verde" (Green Public Procurement) y participa en proyectos europeos que promueven la bioeconomía circular. Como miembro del Biolndustries Consortium (BIC), CTA participa en el diseño de los programas europeos de financiación en bioeconomía de la BioBased Industries Joint Undertaking (BBI JU).

\section{Proyectos de bioeconomía circular donde participa CTA}

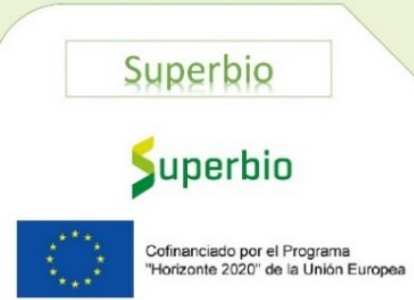

SuperBIO es un proyecto con 10 socios de 6 paises europeos y financiado por el programa Horizonte 2020. SuperBIO ha construido más de 40 nuevas cadenas de valor innovadoras

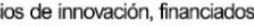
al menos en un $75 \%$, a más de 37 pymes para madurar sus modelos de negocio y dar lugar a cerca de 40 nuevas tecnologias, bioprocesos y bioproductos.

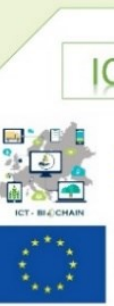

ICT-Biochain

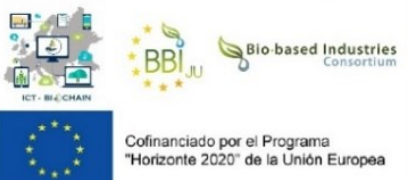

La Consejeria de Agricultura, Pesca y Desarrollo Rural de la Junta de Andalucía y CTA participan, junto a otros 6 socios, en el proyecto europeo ICT-BIOCHAIN, financiado por la iniciativa conjunta BBI JU en el marco de Horizonte 2020, para impulsar la digitalización de las cadenas de suministro de digitalización de las cadenas de suministro de Innovation Hubs, uno de los cuales se ubicará en Andalucía como región piloto.

http://www.h2020-superbio.eu/

\section{https://ictbiochain.eu/}

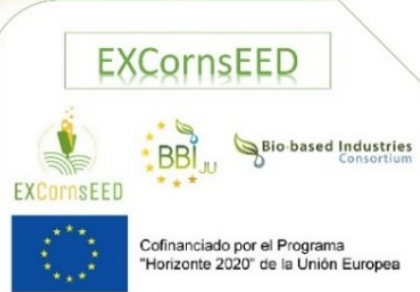

El proyecto EXCornsEED aglutina a 13 socios de 8 paises europeos y cuenta con un presupuesto de 7,2 M€ para desarrollar tecnologias sostenibles que permitan obtener ingredientes funcionales para alimentación, productos químicos y cosmética a partir de productos quimicos y cosmética a partir de
los efluentes secundarios de biorrefinerias de los efluentes secundarios de biorrefinerías de maiz, bioetanol y biodiésel. CTA lidera los
análisis de sostenibilidad y las tareas de explotación de resultados del proyecto.

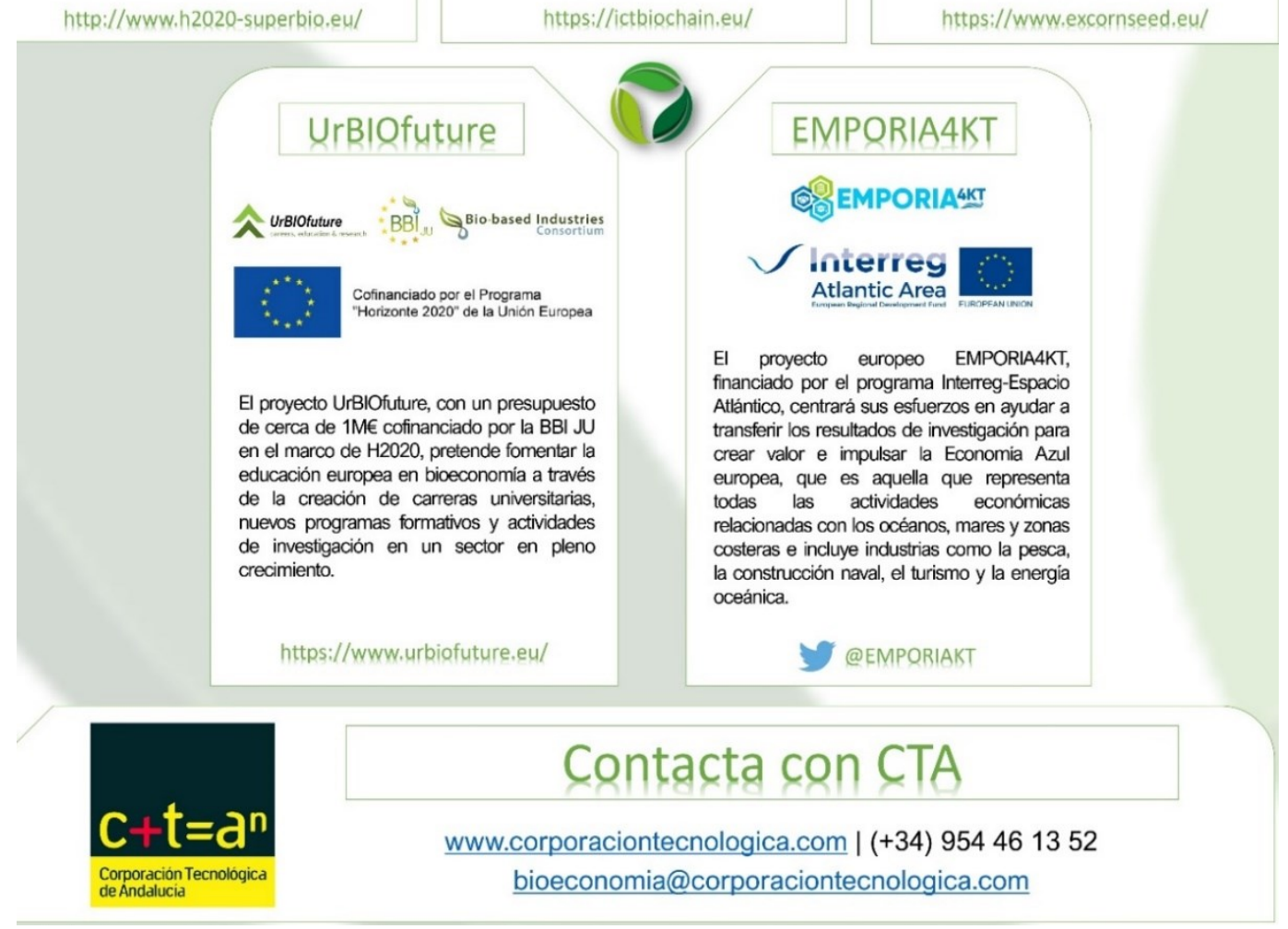




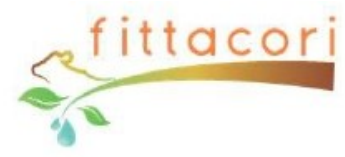

La Fundación para el Fomento y Promoción de la Investigación y Transferencia de Tecnología Agropecuaria (FITTACORI), es el ente financiero del Sistema Nacional de Investigación, Innovación y Transferencia de Tecnología Agropecuaria (SNITTA).

Es una Fundación privada de utilidad pública, sin fines de lucro, que tiene como misión contribuir al desarrollo agropecuario de Costa Rica a través del fomento de la innovación y la transferencia de la ciencia y la tecnología agropecuaria.
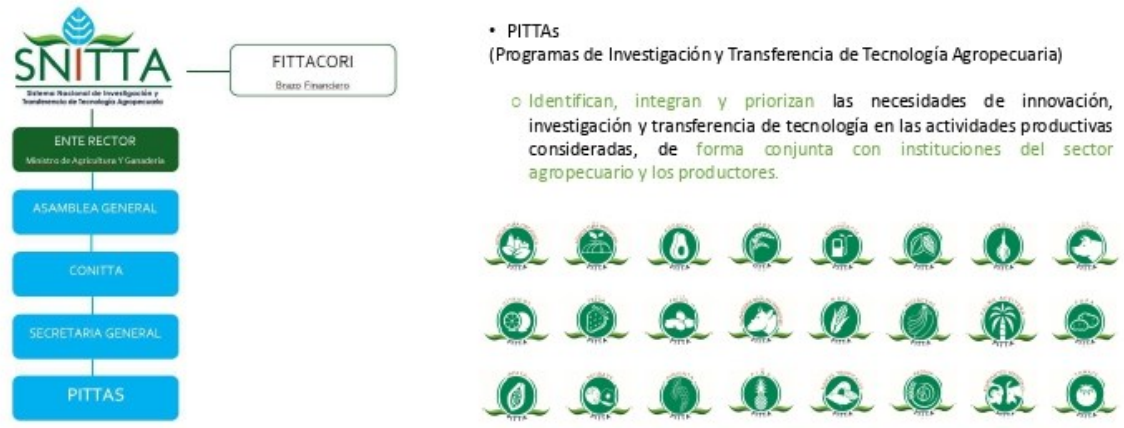

\section{FITTACORI y la bioecconomía}
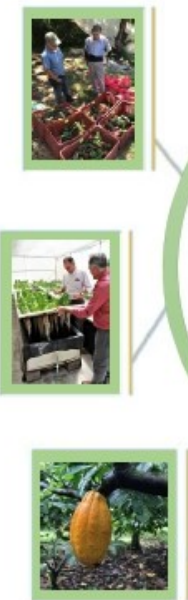

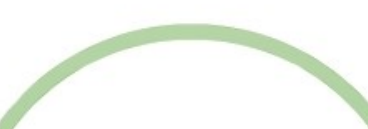

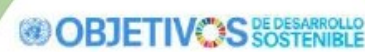

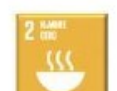

\section{似}

\section{(12:}

C
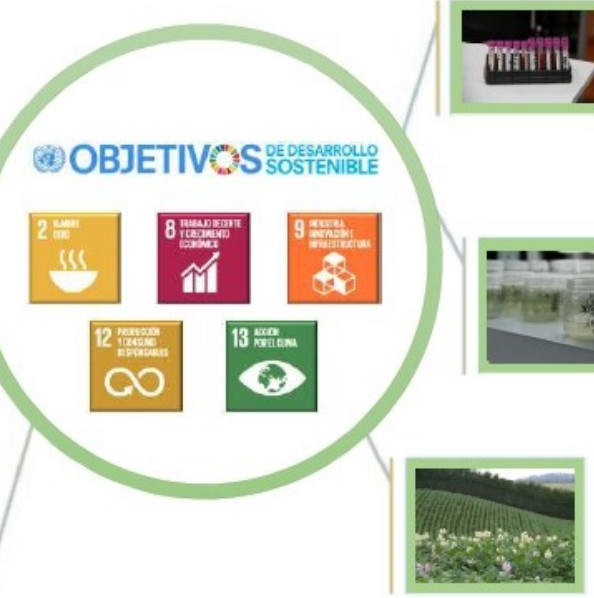

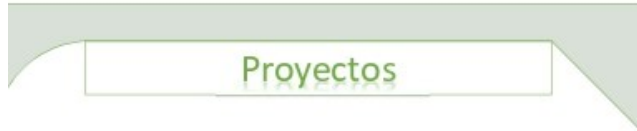

Investigadores(as) y productores(as) de diversas áreas trabajando juntos(as) en más de 90 proyectos agropecuarios.
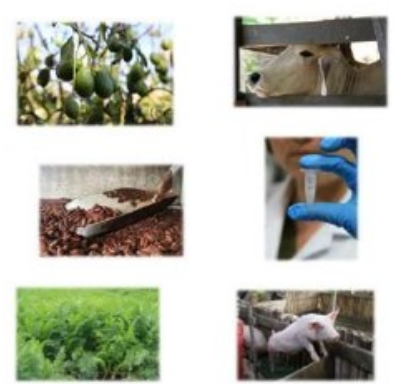

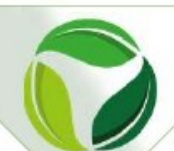

Ti=2

\section{Formación y publicaciones}

$$
\begin{array}{ll}
\checkmark \text { Más de } 42000 & \begin{array}{l}
\text { Productores(as) y técnicos(as) } \\
\text { capacitados(as) }
\end{array} \\
\checkmark \quad \text { Más de } 100 & \text { Publicaciones }
\end{array}
$$

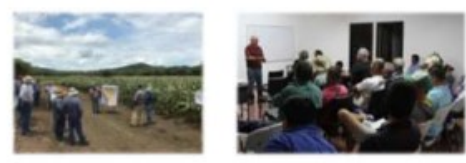

\section{mww. flittacorl.or.cr}

f https://es-la.facebook.com/Fittacori/ Correo: info@fittacori.or.cr

C3-BIOECONOMY, Revista de Investigación y Transferencia en Bioeconomía Circular y Sostenible Nº 12020) 


\section{FUNICA}

La Fundación para el Desarrollo Tecnológico Agropecuario y Forestal de Nicaragua (FUNICA) es una fundación de naturaleza civil, apolitica y sin fines de lucro, con personalidad jurídica y gobierno propio, constituida por 24 instituciones del sector público y privado que incluyen gremios de productores agropecuarios y forestales, universidades, ONG y asociaciones de profesionales de las ciencias agropecuarias. Constituida según escritura No 7 del 01 de Noviembre del 2000 y Decreto legislativo No. 2867 del 5 de Abril del 2001, publicado en la Gaceta No. 71 del 17 de Abril del 2001.

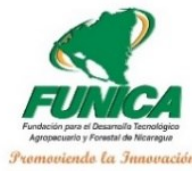

MISION: Contribuir al crecimiento económico inclusivo y sostenible de las Redes Miembro: poblaciones rurales mediante la gestión del conocimiento para ta innovación tecnológica de servicios y procesos.

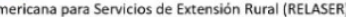

VISION: Ser un referente en la gestión de la innovación agropecuaria y forestal, en
el sector rural. Red INNOVAGRO, Foro Global para los Servicios de Asesoría Rural (GFRAS). Con quienes trabaja FUNICA:

P Productores individuales organizados en cooperativas, asociaciones de productores, técnicos, empresas oferentes de productos y servicios tecnológicos, micro empresas rurales, emprendedoras rurales, grupos de mujeres $y$ jóvenes rurales.

ב Articula alianzas estratégicas con actores de las diferentes cadenas de valor para complementar servicios tecnológicos que promuevan la innovación. Además apoya a otros actores públicos y privados tales como universidades, ONG, centro de formación técnica y profesionales individuales para promover las innovaciones tecnológicas.

\section{Programas y logros}

Programas y acciones:

a Fomento de asistencia técnica, productos y servicios tecnológicos en la cadena de valor, para asegurar que los productores adopten nuevas tecnologias en sus sistemas de producción, transformación y comercialización

$\checkmark$ Emprendimiento rural y desarrollo empresarial, centrado en desarrollar e impulsar la asociatividad, el movimiento de empresas rurales, autogestionarias y eficientes.

Desarrollo institucional de FUNICA y sus asociados, orientado a la consolidación de la Fundación.

Experiencia en implementación de proyectos y programas:

La fundación ha invertido fondos para el mejoramiento de capacidades productivas $y$ de mercado de pequeños $y$ medianos productores (as). Promover el mercado de
tecnologias en los oferentes y demandantes, gestión del conocimiento. La finalidad es contribuir al mejoramiento de sus ingresos y el nivel de vida de familias en condiciones climáticamente adversas con equidad de género. Todo esto con el apoyo de las organizaciones como: Fondo Internacional de Desarrollo Agricola (FIDA), Agencia Suiza para el Desarrollo y la Cooperación (COSUDE), Cooperación al Desarrollo de Dinamarca (DANDA), Reino de los Paises Bajos de Holanda, Fundación Mundial, IICA, Rainforest Alliance/UTZ, CAINCO-Unión Europea

\section{Logros obtenidos:}

L Las familias apoyadas por FUNICA han logrado cambios en su condición de vida,
mejorando su autoempleo y sus ingresos económicos.

- Aportes sustanciales en conocimiento para la construcción de politicas y .

- Construcción de una plataforma de diálogo y consenso que articula a los principales actores del sector.

$\checkmark$ Consolidación del mercado de servicios y tecnologías.

- Articulación del sistema de investigación agropecuaria y forestal a la extensión.

Incremento de la oferta de diferentes servicios de asistencia técnica, empresariales $y$ tecnolód
familias nicaragüenses.

Disponibilidad de tecnologias amigables con el medio ambiente, a bajos costos $y$

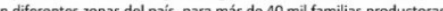

\section{Productos y servicios}

$\checkmark$ Ofrecemos incentivos para desarrollar el mercado de servicios tecnológicos a los de proyectos y planes de negocios.

च Servicios de desarrollo empresarial rural a través del Centro de Gestión e Innovación empresarial (CGIE-FUNICA); analisisis técnico, económico y financiero de rubros conologia.

ב Asesorias de expertos especializados en temas de sistemas de innovación agricola, prospección tecnológica, empresarial y de negocios

Promovemos la conformación de alianza y redes para asegurar el abordaje integral de los rubros agropecuarios

\lrcorner Desarrollamos capacidades para la investigación agropecuaria y forestal; estudios de mercado de productos y servicios agropecuarios, sectoriales y sub sectoriales para

- Asistencia a través de la Escuela de Negocio; Asistencia técnica para producción y comercialización en rubros agropecuarios.

] Fomentamos el desarrollo empresarial a cooperativas y emprendedores Fes

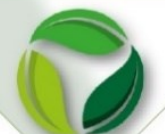

\section{Publicaciones}

Nicaragua; Tecnología Agropecuaria. Su rol en una politica del sector agropecuaria

La demanda manda. Igual de oportunidades de género en el fondo de Asistencia Téenica.

Evolución del Fondo de Asistencia Técnica en Las Segovia 2004-2009

Fondo de Asistencia Técnica. Experiencias de la primera fase y futuras acciones

Para combatir el hión básica de salud de plantas. implementación del Bono Productivo Alimentario en el occidente de Nicaragua.

Estudio de Impacto de los servicios de asistencia técnica en el occidente del

Servicios y tecnologías agricolas en Las Segovia: una valoración externa del estado actual.

Experiencias en el desarrollo de mercados locales de tecnologías agropecuarias.

te para la formulación de planes de negocios de iniciativas de Tecnologías Agropecuarias.

(acropecuarias y Forestales.

las experiencias de FUNICA en el desarrollo de mercados locales de tecnologías 2006-2008

- Contacto www. funica.org.ni, correo electrónico: comunicacion@funica. org.ni 


\section{0 \\ C3-BIOECONOMY}

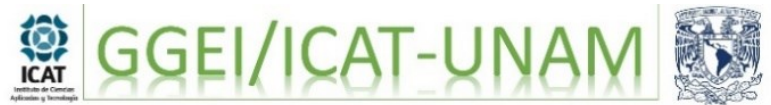

El Grupo Gestión Estratégica de la Innovación (GGEI) forma parte del Instituto de Ciencias Aplicadas y Tecnologia (ICAT) de la Universidad Nacional Autónoma de Mexico (UNAM). Liderado por los doctores José Luis Solleiro y Rosario Castañón. el GGEl tiene por objetivo desarrollar metodologias, modelos y anáüis de casos, entre otras contribuciones académicas, originates y relevantes para ta gestionn estrategica de la innovación en diferentes ambientes institucionates, asi como promover la apticacion de los conocimientos generados a través de la prestación de servicios especializados y la formación de recursos humanos en diversos sectores.

\section{(C) Integrantes del grupo}
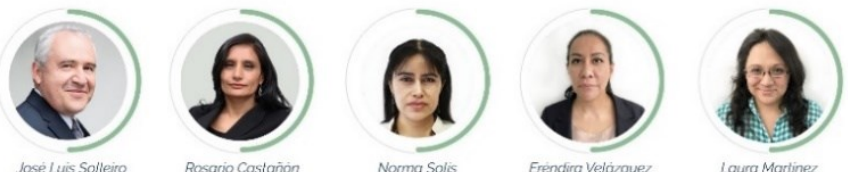

Lineas de investigación

- Politicas de innovación

- Gestión de transferencia de tecnologia

Sistemas regionales de innovación

- Vinculación universidad-empresa

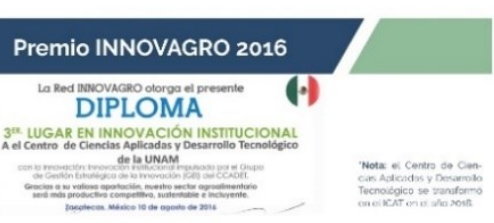

\section{GGEI y la bioeconomía}

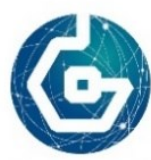

Grupo Gestión

Estratégica de la Innovación

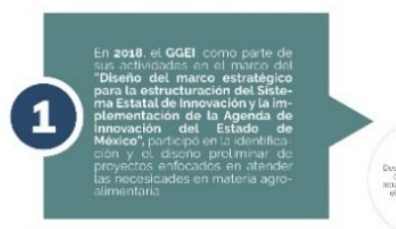

3)

\section{표 $=$}
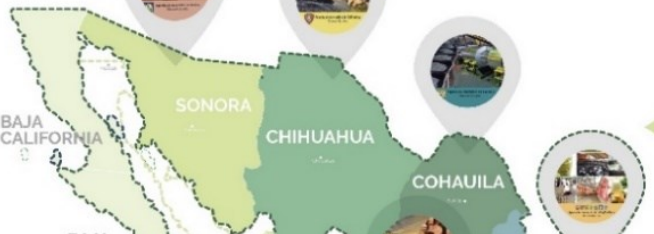

(1) Escanea estos codigos oR y tendras las

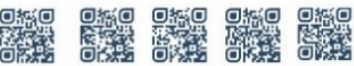
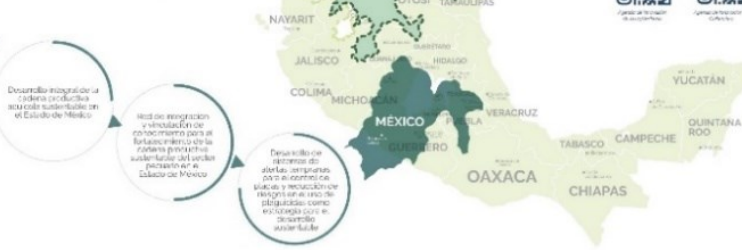

Formación x publicaciones

\section{Proyectos e iniciativas}

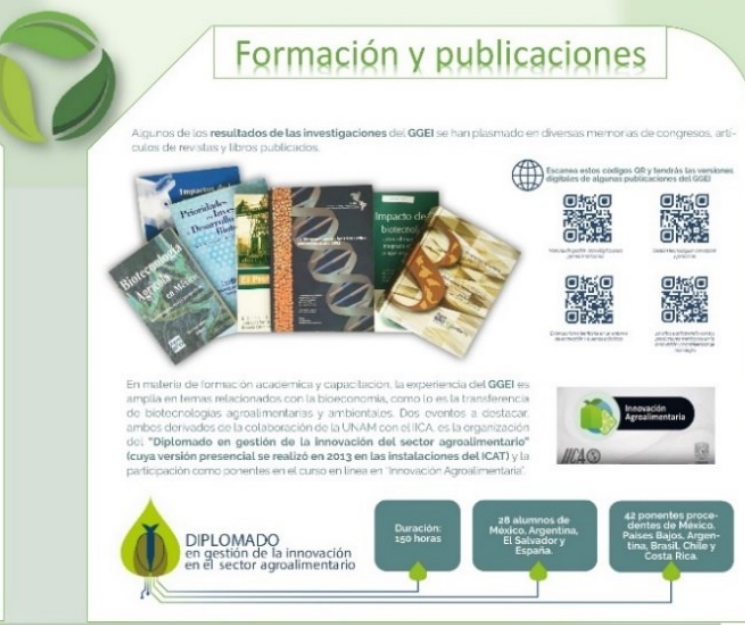

C3-BIOECONOMY, Revista de Investigación y Transferencia en Bioeconomía Circular y Sostenible $N^{\circ} 1$ (2020) 

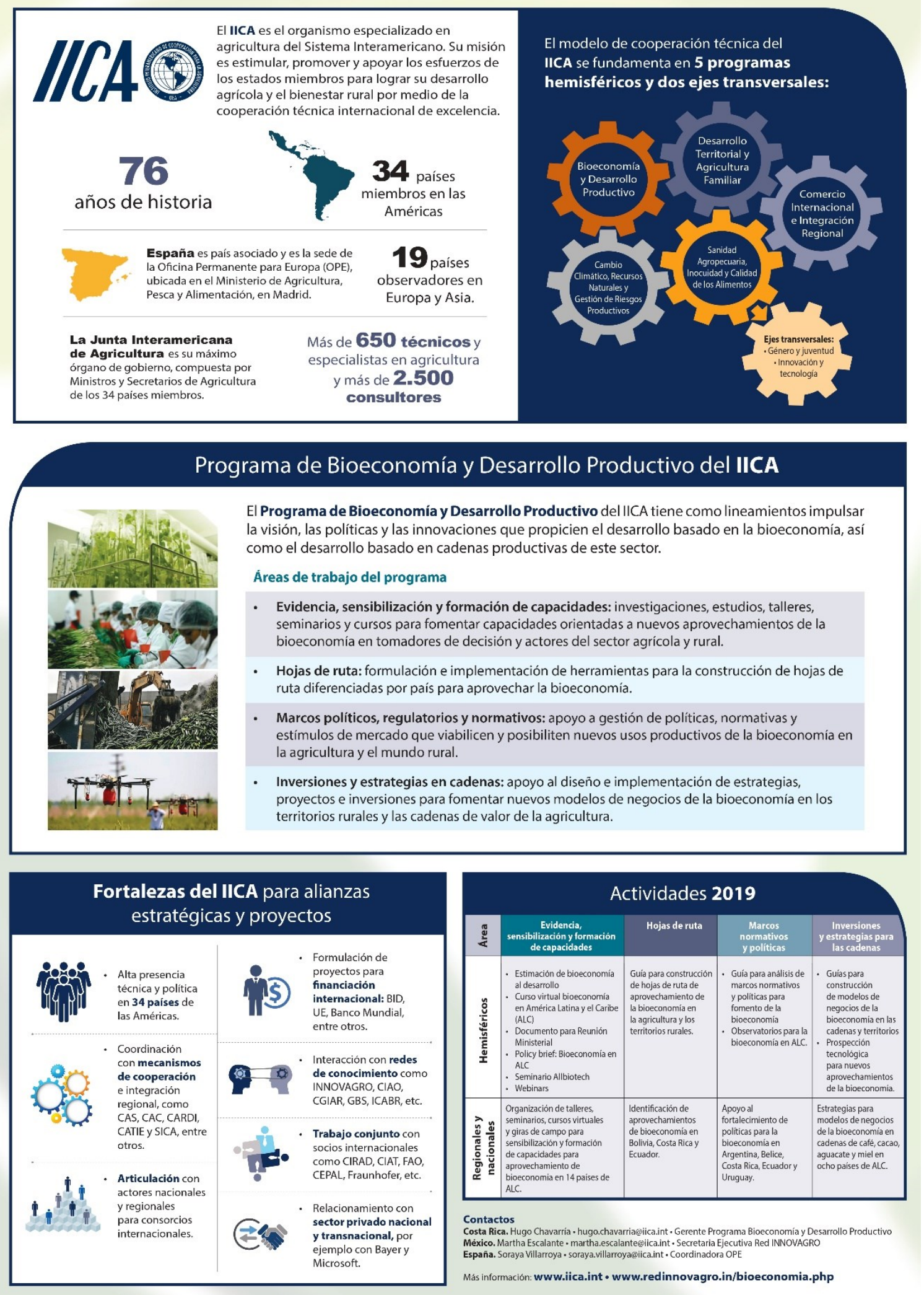


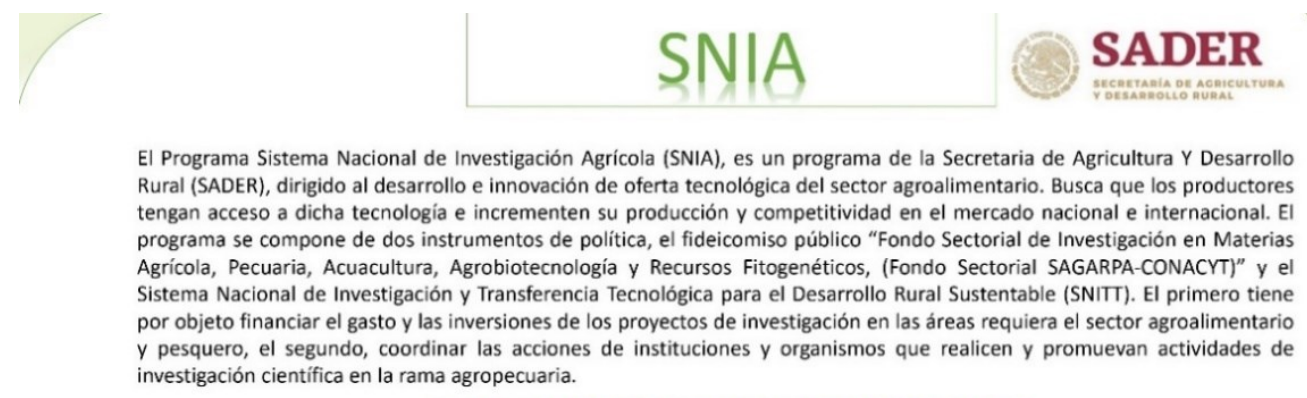

\section{PRINCIPALES UNIVERSIDADES Y CENTROS DE} INVESTIGACIÓN
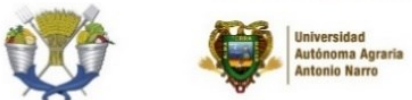

(1) $\begin{aligned} & \text { Colegio de } \\ & \text { Postgraduados }\end{aligned}$

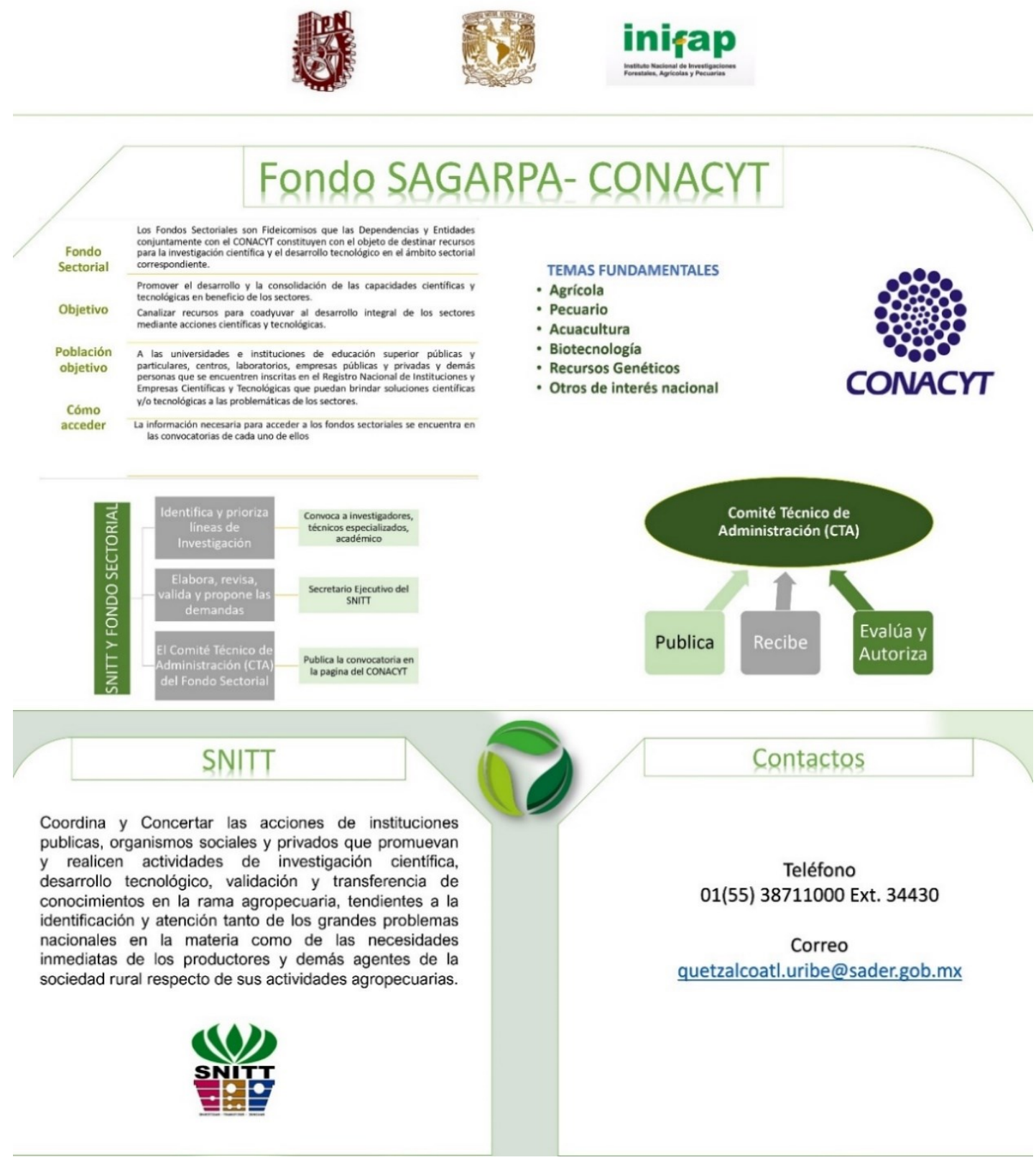

C3-BIOECONOMY, Revista de Investigación y Transferencia en Bioeconomía Circular y Sostenible N¹ (2020) 


\section{Centro Tecnnológico Tecnnova}

La Fundación para las Tecnologías Auxiliares de la Agricultura es el Centro Tecnológico de la Industria Auxiliar de la Agricultura, la Postcosecha y el Envasado, aportando valor, a través de la

innovación y la tecnología, a la cadena de valor de las frutas y hortalizas, desde la producción hasta la tecnología post-cosecha y transformación de alimentos.

- Se creó en 2001.

- Centro Tecnológico Andaluz desde 2007

- Centro Tecnológico Nacional desde 2015.

- Agente local de la Red PIDI perteneciente al CDTI.

- Oficina de proyectos europeos.

- Entidad de Transferencia de la Tecnología y el Conocimiento Andaluza

- OTRI no registro 236 reconocido por Ministerio de Economía y Competitividad.

Conforma un Cluster de la agroindustria, con diferentes agentes del sector y 105 empresas de los diferentes subsectores de la Industria Auxiliar de la Agricultura, que aportan innovaciones a la cadena de valor agroalimentaria.

\section{CT Tecnova y la bioeconomía}

\section{CT TECNOVA ofrece al sector:}

- Un equipo de 25 investigadores especializado.

- Involucración en iniciativas internacionales en el marco de la bioeconomía y contacto con los principales agentes europeos.

- Instalaciones para la I+D+i y el desarrollo de cadenas de valor en la bioeconomía:

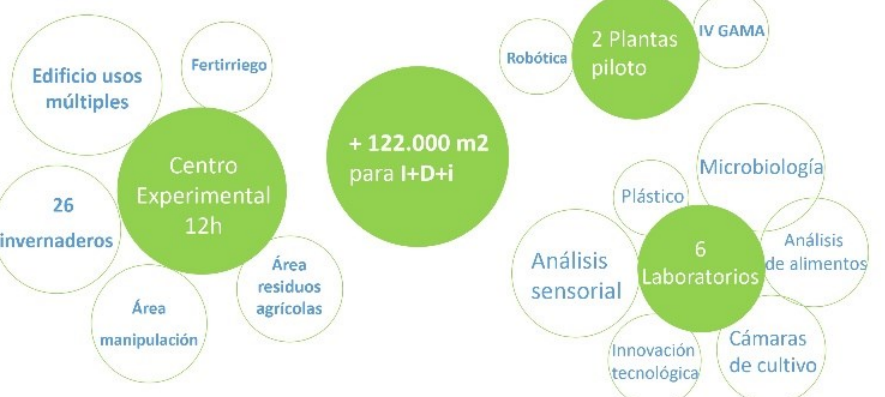

Miembros del Cluster de Bioeconomía Andaluza

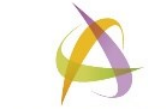

estrategia andaluza de bioeconomia circular

\section{Proyectos e iniciativas}
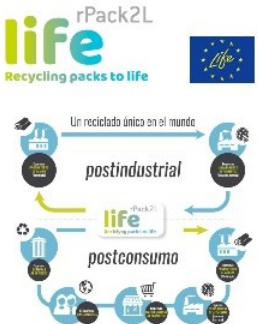

GRUPO OPERATIVO
SUPRA-AUTONOMIICO:

AgriREFIN

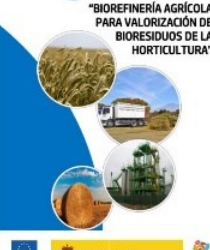

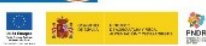

Proyectos e iniciativas

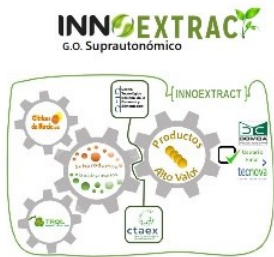

tis:

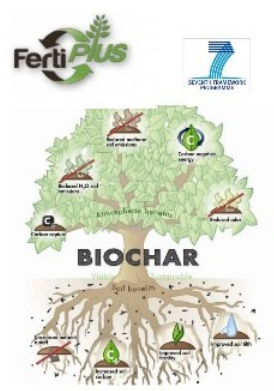




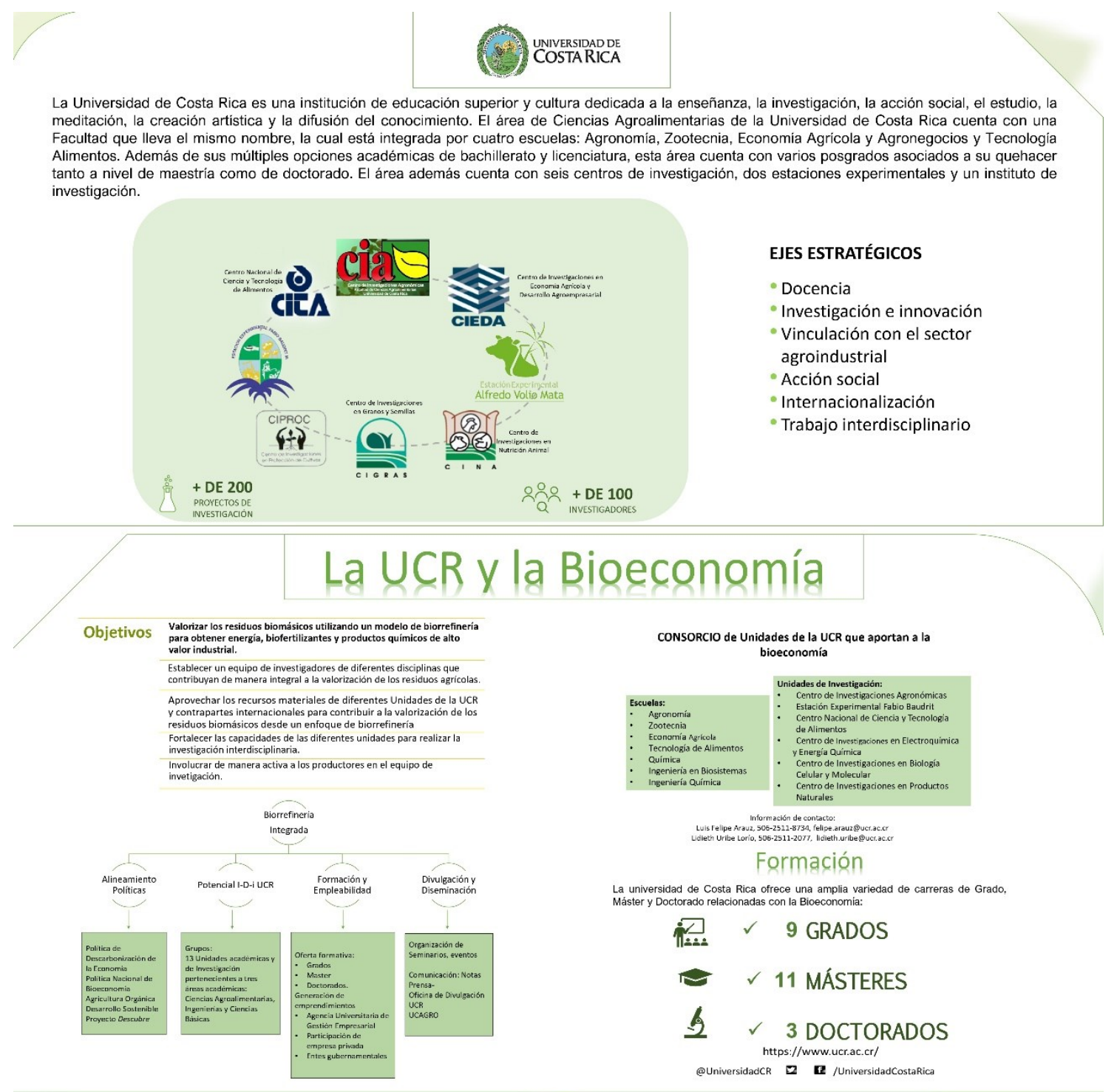

Proyectos e iniciativas

Valorización de residuos de cultivos tropicales bajo el concepto de biorefinería integrada en la Universidad de Costa Rica

La Universidad de Costa Rica posee una importante capacidad cientifica y laboratorios de punta en diferentes Escuelas y Centros de Investigación. Sin embargo los esfuerzos para valorizar la biomasa residual de la agricultura han sido aistados lo cual no ha propiciado ha inserion eficiente en el tema de residuos agricolas orgánicos (RAO) principalmente: caña de azúcar, piña, palma nja, ganadería de leche y carne, avicultura y porcicultura. Un enfogue de biorrefinerla integrada pern obtención de energía y productos químicos de interés industrial a partir de dicha biomasa residual, contribuyendo a la reducción de la producción de gases de efecto de invernadero y abriendo la oportunidad a nuevos emprendimientos basados en materiales subutilizados

para uling piña, el café $y$ del arroz químicos de alto valor industrial, aprovechando la capacidad instalada en ta Universidad de Costa Rica.

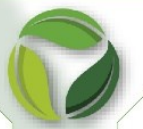

La estrategia; Biorrefinería Integrada

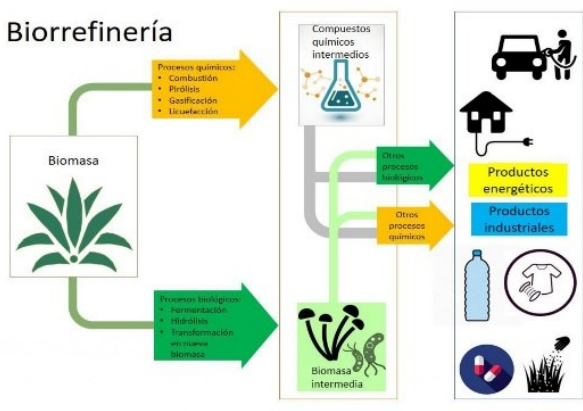


La Universidad Nacional Agraria La Molina (UNALM) es una institución educativa universitaria especializada en la formación de profesionales competentes para los sectores agrosilvopecuario, pesquero, alimentario y económico. La UNALM, pretende alcanzar el nivel de liderazgo, basado en la enseñanza-aprendizaje, investigación, extensión universitaria y proyección social, de calidad, y ser referente en los sectores agrosilvopecuario, pesquero, alimentario y económico, promoviendo el manejo de los recursos naturales y la conservación del ambiente para el desarrollo del país.

La UNALM, adicionalmente de participar en la

Red Innovagro, también forma parte de la RED

RED DE INVESTIGACIÓN,

IDi, asociación de universidades peruanas DESARROLLO E INNOVACIÓN

que conecta ciencia y tecnologia, con el sector RED IDi

empresarial y gubernamental

\section{UNALM y la Bioeconomía}

-Asegurar la calidad de la formación profesional de los estudiantes de pre y posgrado

-Fortalecer la Investigación

Objetivos desarrollo e innovación en la UNALM

- Fortalecer la extensión universitaria y la proyección social en la UNALM

- Fortalecer y modernizar de la gestión institucional
LINEAS DE INVESTIGACIÓN

*Economía de la Innovación agricola, desarrollo rural y seguridad alimentaria

* Economía ambiental, de los recursos naturales y del cambio climático

* Economía y aprovechamiento forestal

* Materia prima y productos terminados procedentes de bosques naturales

y plantaciones forestales

* Sistemas de producción agrícola sustentable

* Innovación empresarial agrícola

* Procesos de transformación quimica de los productos forestales maderables y no maderables

* Tecnología para la pequeña agricultura

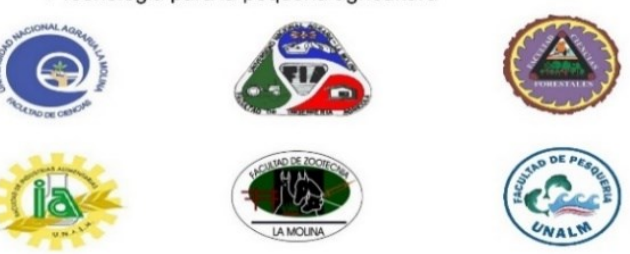

\section{Proyectos e iniciativas}

Investigadores de la UNALM están participado en numerosos proyectos nacionales e internacionales en el ámbito de la Bioeconomia que avalan el potencial del campus en la materia. A continuación se citan algunos de los proyectos vigentes de UNALM:

$\checkmark$ Valorizando la biodiversidad del Peri

$\checkmark$ Evaluación de las existencias comerciales y estrategia para manejo sostenible de la caoba (Swietenia macrophylla) en Peri.

Aprovechamiento sostenible de los hongos pigmentadores de madera para su uso comercial en el desarrollo de artesanías turismo vivencial en Madre de Dios.

Obtención de biocombustibles sólidos mediante los procesos de torrefacción y densificación de biomasa residual de caballerizas con alto contenido de cascarilla de arroz (Oryza sativa).

$\checkmark$ Utilización de la técnica de dobles haploides en el aprovechamiento de la biodiversidad del maiz peruano para su uso como alimento nutracéutico.

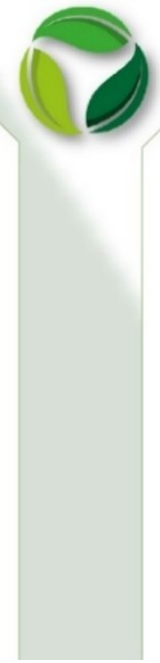

Formación y publicaciones

Las UNALM oferta una amplia variedad de formación de Grado, Máster y Doctorado con relación a la Bioeconomia:

$V_{i=1} \quad \checkmark 5$ GRADOS

$\checkmark 11$ MÁSTERES

\& $\checkmark 3$ PROGRAMAS DE DOCTORADO

uww.lamolina.edu.pe

$f$ You Tube 


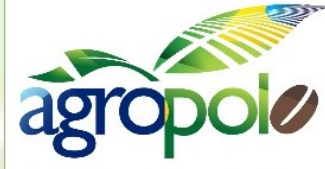

CAMPINAS・BRASIL

\title{
Innovación en Bioecconomía en Brassil
}

Agropolo Campinas-Brasil se creó en junio de 2015, en un esfuerzo por desarrollar un ecosistema de innovación de clase mundial con énfasis en Bioeconomía en la Ciudad de Campinas, Brazil. Durante el período 2015-2018 las actividades del Agropolo se concentraron en el desarrollo de un "roadmap tecnológico" para Bioeconomía Tropical en 12 áreas estratégicas. Se elaboró un Proyecto de Políticas Públicas en Bioeconomía - PPPBio, apoyado por la Fondación de Apoyo à la Investigación del Estado de São Paulo (FAPESP), con el objetivo de guiar el desarrollo de la Bioeconomía en São Paulo.

\section{Conectando Personas \& Associando}

Agropolo Campinas-Brazil es una plataforma interinstitucional basada en conceptos de Innovación Colaborativa

Como una nueva estrategia para promocionar la investigación, el desarrollo tecnológico, y productos y servicios innovadores. La plataforma será construida para incrementar las conexiones entre las instituciones de educación superior e investigación y el sector privado, resultando en una investigación diferenciada por proveer mejores oportunidades de empleo y rentabilidad para las actividades de Bioeconomía

\section{Gobernanza \& Asociación}

\author{
SÃO \\ IAC ITSL

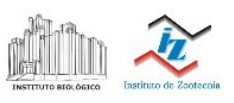 \\ Fundadores \\ GOVERNODOES \\ Embrapa \\ Parcerías Técnicas y Apoyos \\ Stakeholders \\ $y$ tfolland.

\section{2 Áreas Estratégicas en Brașil}

\section{- RESIDUALES agrícolas y urbanos} \\ - AGRICULTURA DE PRECISIÓN \\ - ACEITES ESENCIALES, AROMÁTICOS y PLANTAS MEDICINALES \\ - Sistemas de PRODUCCIÓN ANIMAL \\ - Uso sustentable del AGUA \\ - Nueva Industria: CAFÉ y CITRICOS \\ INGREDIENTES y Alimentos PROCESADOS FUNCIONALES \\ - PAQUETES para Alimentos y Bebidas \\ - TECNOLOGÍAS DE PROCESAMENTO para Alimentos y Bebidas \\ - BIOCOMBUSTIBLES Avanzados \\ - Biomasa para QUÍMICOS \\ - ENZIMAS \& QUÍMICA VERDE
}

SÃO

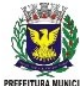
Nerivativern UNICAMP IICHe AGR POLIS GOVERNODOES

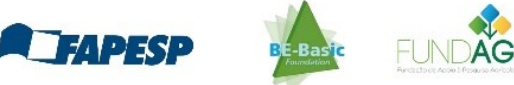
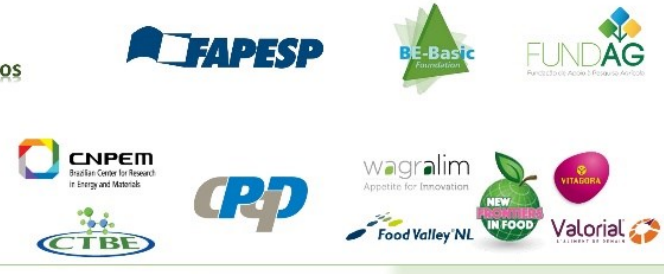

\section{BIOECONOMÍA TROPICAL}

Roadmap Tecnológico y

Directivas para el Resarrollo de

la Bioconomía en São Paulo-

Brasil

EN CONSTRUCCIÓN,

(Octubre, 2019)

Contacto

www.agropolocampinasbrasil.org agropolo@agropolocampinasbrasil.org +55192137.0621 


\section{Wageningen University \& Research (WUR)}

“Explorar el potencial de la naturaleza para mejorar la calidad de vida” Ésta es la misión de Wageningen University \& Research (Universidad y Investigación). Contamos con 6.500 trabajadores y 10.000 estudiantes de aproximadamente 100 países que trabajan bajo nuestra supervisión sobre alimentación saludable y medio ambiente en todo el mundo ya sea para los gobiernos como para las empresas privadas. El poder de Wageningen University \& Research radica en la fusión de los gobiernos como para las empresas privadas. El poder de Wageningen University \& Research radica en la fusión de como naturaleza, tecnología y ciencias sociales. De ésta manera se logran los avances científicos de una manera rápida en la práctica y se traduce en la educación. Éste es el enfoque de la Universidad de Wageningen.

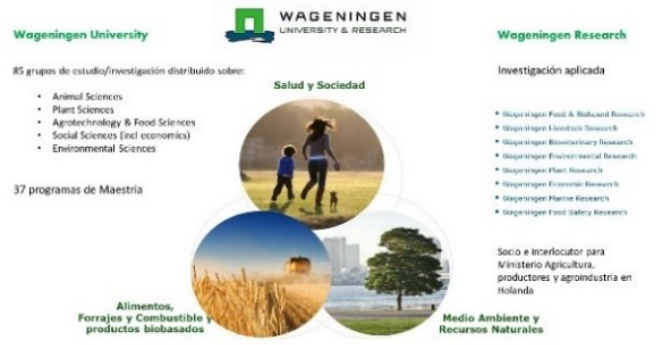

La especialidad de la universidad de Wageningen radica en las siguientes tres áreas centrales relacionadas:

- Alimentos, forrajes, combustibles y productos biobasados

- Medio ambiente

- Salud, estilo y condiciones de vida

\section{WUR y la bioecconomía}

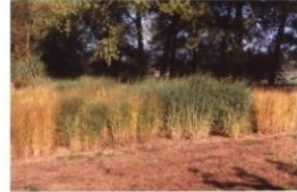

Temas de Investigación :

Potenciales de biomasa y movilización en sistemas terrestre y acuatico

sa para el mercado

plantas para aplicaciones

- Uso de tierra sostenible y la producción de biomasa para nutrición, biomateriales y

bioenergía

- Combinaciones y compensaciones de producción

de biomasa con mitigación de gases efectos invernaderos y proteccion de biodiversidad

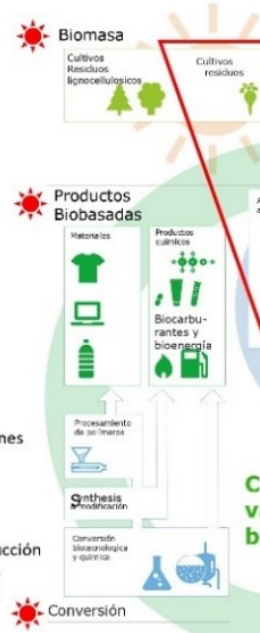

Proyectos e iniciativas

Investigadores y universidades del WUR han participado en numerosos
proyectos nacionales $\mathrm{e}$ internacionales en el ámbito de la Bioeconomia que

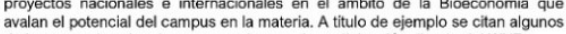
de los proyectos vigentes qus enentan con la participación directa del Wur.

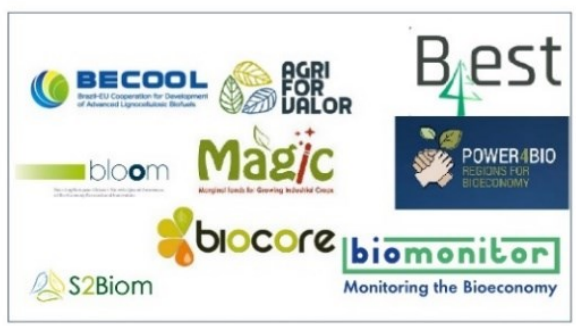

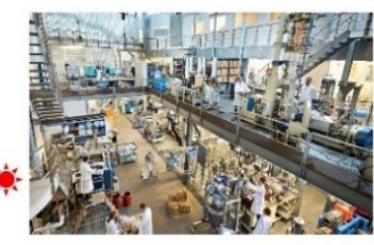

Llevar resultados de lab a piloto

Temas de Investigación :

- Biorefineria \& cadenas sostenibles

- Químicos \& combustibles biobasados

- Nuevas fuentes de proteínas

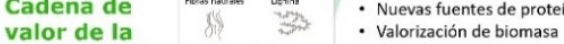
bioeconomía
28

Componentes de
biomasa

:

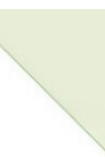




\section{REFERENCIAS}

Bioeconomy: the European way to use our natural resources. Action Plan 2018. Recuperado de: https://ec.europa.eu/research/bioeconomy/pdf/ec_bioeconomy_bo oklet_2018.pdf\#view=fit\&pagemode=none.

FAO: Retos y oportunidades en un mundo globalizado. (2019). Recuperado de: http://www.fao.org/3/ca4305es/ca4305es.pdf.

IICA: Plan de Mediano Plazo 2018 - 2022. Recuperado de: http://repositorio2.iica.int/handle/11324/7191

Red Innovagro. Memoria del IX Encuentro: Bioeconomía y ecosistemas de innovación.

Disponible en: https://www.redinnovagro.in/pdfs/ENCUENTROINNOVAGRO2019.pdf. 